\title{
Vitamin B 12 Assay - are we on Track?
}

Rateesh Sareen $^{1 *}$, G N Gupta ${ }^{2}$

${ }^{1}$ D.N.B Pathology, Department of Pathology \& Transfusion Medicine, Santokba Durlabhji Hospital, Jaipur.

${ }^{2} \mathrm{Head}$ of department, Department of Pathology \& Transfusion Medicine, Santokba Durlabhji Hospital, Jaipur.

Corresponding Author: Rateesh Sareen, D.N.B Pathology, Department of Pathology \& Transfusion Medicine, Santokba Durlabhji Hospital, Jaipur, India.

Received date: September 23, 2020; Accepted date: October 01, 2020; Published date: October 06, 2020

Citation: Sareen R., G N Gupta, (2020) Vitamin B 12 Assay - are we on Track? J, Clinical Medical Reviews and Reports. 2(8); DOI:10.31579/2690-8794/045

Copyright: ( ) 2020, Rateesh Sareen, This is an open access article distributed under the Creative Commons Attribution License, which permits unrestricted use, distribution, and reproduction in any medium, provided the original work is properly cited.

\section{Abstract}

Vitamin B12 assay is one of the most frequently ordered tests particularly as a part of regular medical checkups. The deficiency is rampant in vegetarian population. It is of immense importance that laboratories establish their own reference interval (RI) of analyte specially Vitamin B12 as a diagnosis of Vitamin B12 deficiency based on RI of kit insert inadvertently leads to unnecessary treatment or work up. A blind reliance on RI of kit insert should be discouraged as they do not take into account population characteristics and do not truly reflect RI specific to the population under study.

Key words- vitamin B12, reference interval (RI), laboratories

\section{Introduction}

The water soluble Vitamin B12 is required for red blood cell formation, DNA synthesis and neurological function in human body exists in two active forms methycobalamin and 5-deoxyadenosylcobalamin [1,2, and $3]$.

Dietary Vitamin B12 is bound to protein and therefore requires release by action of hydrochloric acid and protease enzyme in the stomach [3]. The synthetic form of Vitamin B12 is a free form that does not require metabolism in stomach. It directly combines with the intrinsic factor and gets absorbed within distal ileum by receptor mediated endocytosis [4].

Vitamin B12 is assessed by serum or plasma levels and values below 175$250 \mathrm{pg} / \mathrm{ml}$ for adults are indicative of Vitamin B12 deficiency [3].

The literature evidence suggests that serum Vitamin B12 levels might not precisely reflect intracellular concentration [5]. Even the surrogate markers like homocysteine levels (value $>13$ micro mol/L) [6] and methyl malonic acid levels (value $>0.4$ micro $\mathrm{mol} / \mathrm{L}$ ) have poor specificity [4]. The laboratory diagnosis of Vitamin B12 status is based on mathematical model that combines multiple markers of Vitamin B12 status into a single diagnostic indicator a four variable analysis to calculate a combined indicator of vitamin B12 status [7].

$$
\text { Log } 10 \text { (Holotranscobalamin X Serum Vitamin B12) X Age factor }
$$

Vitamin $B 12$ status $=$

Methyl malonic acid $\mathrm{X}$ Homocysteine level

This formula transforms all four markers to single variable dependent on age and is called Fedosov's wellness score. It has been recently used in Non-anemic healthy Swiss citizens [8].

In India with large number of population having vegetarian diet and with limited resources, cost constraints it is not possible to apply Fedosov's wellness score. Majority of patients are screened by complete blood count parameters including MCV.

The International Federation of Clinical Chemistry mandates every laboratory to have their own reference limits [9]. But unfortunately most of the laboratories in India follow reference intervals established in western population. The reference interval can be seriously factitious considering the variation in Indian and Western populations due to changes in dietary habits, life style and other factors. There is paucity of literature with regard to reference interval of Vitamin B12. The study aims to establish the reference interval (RI) for Vitamin B12 in our own population.

\section{Material and methods}

We measure Vitamin B12 levels by VITROS ${ }^{\circledR}$ Chemiluminesce Detection system (Micro Well Technology) Ortho Clinical Diagnostics U.S. The assay uses calibrators traceable to the certified National Institute of Standards and technology reference interval. The retrospective data was analyzed after ensuring that daily quality control was acceptable on each day of testing. The study included all patients who underwent Serum Vitamin B12 concentration over past one year. The exclusion criteria were:

- All minors < 18 years of age

- Hemoglobin $<12.6 \mathrm{~g} / \mathrm{dl}$ (Both male \& female)

- Hemoglobin Males $>16.5 \mathrm{~g} / \mathrm{dl} \&$ Females $>16.0 \mathrm{~g} / \mathrm{dl}$

- Mean corpuscular volume (MCV) < 83fl and >101fl

- WBC- Less than $4000 / / \mathrm{mm} 3$ and more than $10000 / / \mathrm{mm} 3$

- Platelet count less than $1,40,000 / / \mathrm{mm} 3$ and more than 4 , $50,000 / / \mathrm{mm} 3$

The complete blood count was analyzed on Sysmex XN1000 hematology 6-part auto analyzer.

The prime objective of the study was to evaluate RI in adult population in western India thriving predominantly on vegetarian diet. A total of 7416 patients underwent Vitamin B12 assay estimation over one year period in 
the year 2018. After applying the exclusion criteria 2510 patients with normal Mean corpuscular volume (MCV) were studied to determine the reference interval of Vitamin B12 in Indian population.

The analysis showed that Vitamin B12 and MCV do not show statistically significant correlation over entire 7416 patients. $(r=-0.07361, p<0.0001$ significant). Statistical analysis was done using Medical (online free trial version) to check normality by Shapiro-Francia test and calculation of reference interval done.

The double sided reference interval study of serum Vitamin B12 for all adults is summarized in table-1. The lower limit of RI was 159 and upper limit 848.50. We used non parametric log transformation method as the data was not normal having kurtosis and skewness.

\section{Limitation}

- History of intake of VitaminsB12 was not available

- $\quad$ Study is limited to patient seeking medical advice at tertiary care hospital.

- History of drugs, alcohol which may have profound effect on Vitamin B12 level was not available.

\section{Strength}

- $\quad$ Large sample size- Drug supplements and other confounding factors are minimized due to larger sample size

- $\quad$ Proper statistical analysis CLSI guidelines

- Exclusion criteria

- $\quad$ Age old dictum of MCV as surrogate marker of Vitamin B12 deficiency is used as one of the exclusion criteria

\section{Discussion}

Reference interval (RI) plays a pivotal role in clinical laboratory test interpretation and patient care because $80 \%$ of the medical decisions are based on laboratory test results [10]. The original concept of RI was first enunciated by Grasbeck and Saris in 1969 [11]. RI are the soul of laboratory tests because isolated laboratory test values do not have any importance without age and sex specific reference range. Clinical and laboratory standards constitute approved guideline (CLSI) that elaborately described methodology for estimation of RI [12]. Reference interval (RI) as defined by Ceriotti, "is an interval that, when applied to the population serviced by the laboratory correctly includes most of the subjects with characteristics similar to reference group and excludes the others [13]. The majority of RI used today is with reference to population targeting 95\% confidence limits. It implies that 5\% of the results from healthy population will fall outside the reported RI. Therefore, no RI is absolutely right or wrong per se. Most of the laboratories do not establish their own reference range and through fait accompli use the manufacturer's RI provided to them.

Whenever a laboratory introduce a new test in their menu, the accreditation bodies make it mandatory to verify or establish performance characteristics. The new protocol $\mathrm{C} 28-\mathrm{A} 3$ allows laboratories to validate reference interval with small number of samples. A laboratory can now use 20 samples for validating its RI. In case the number of outliers is less than 2 , it is statistically valid for the laboratory to adopt the RI as their own. In case the number of outliers is more than 2 that are present outside RI range then another set of 20 samples must be collected.

The RI (normal range, reference range) to be calculated by using any of the three methods 1) Normal distributed values, 2) Non parametrical percentile method and 3) Robust method. When the sample size is small (less than 20) robust method can be applied. For bigger sample size, the choice of method depends on the nature of distribution of data. Data which has high kurtosis and skewness are best examined by percentile method.
The first and foremost step is to test for outlier based on tests by Reedetal [14] or Tukey [15]. The tests detected outliers which have to be inspected by the investigator so that they are excluded from calculation. The next step is the test for normal distribution. If $p$ value is higher than 0.05 , it may be assumed that the data follows normal distribution hence 'accept' normality. If $\mathrm{p}$ value is less than 0.05then the hypothesis of normal distributed data is rejected. If the data does not have normal distribution it requires logarithmic transformation (positively skewed data). The results of our study are enumerated in underlying tables.

\begin{tabular}{|c|c|}
\hline $\mathrm{N}$ & 2510 \\
\hline Prevalence & $1244 / 25.10 \times 100=49.56 \%$ \\
\hline Skewness & 0.888 \\
\hline Kurtosis & 0.08679 \\
\hline Shapiro test & $\mathrm{W}=0.9022$ \\
\hline \multicolumn{2}{|c|}{ Data not normally distributed } \\
\hline Non Parametric percentile method Log transformation \\
\hline Lower limit & 159 \\
\hline Upper limit & 832.6267 to 869.00 \\
\hline Reference interval & 159 to 843.4996 \\
\hline \multicolumn{2}{|c|}{ Table-1: summary statistics } \\
\hline
\end{tabular}

The prevalence of Vitamin B12 deficiency based on our data was $49.56 \%$ .Our calculated RI for adult came out to be $159-843.5 \mathrm{pg} / \mathrm{ml}$, whereas the kit insert RI is 239-931 pg/ml. It signifies that the Vitamin B12 levels in our population are far low as compared to those of kit insert. Furthermore as the lowest detection limit of the instrument Vitros 3600 was $159 \mathrm{pg} / \mathrm{ml}$ it is possible that it could be further go down in case the detection limit is lower. More studies are required to further elaborate on the lower detection limit. The upper normal reference limit was low in our study from $931 \mathrm{pg} / \mathrm{ml}$ to $843.5 \mathrm{pg} / \mathrm{ml}$.

It is evident that on following the kit insert reference range there is over diagnosis of Vitamin B12 deficiency. Even if we go by mayo clinic RI 180-914ng/l, the kit insert RI was much higher. Considering the limitation of our analyzer the lower limit of normal RI could be further below 159 $\mathrm{ng} / \mathrm{l}$.

\section{Conclusion}

The study established the rationale of using laboratory's own reference interval by laboratories which are calculated on the basis of local and regional demographics.

\section{References}

1. Herbert V. (1996). Vitamin B12 in Present Knowledge in Nutrition. 17th ed. Washington, DC: International Life Sciences Institute Press.

2. Herbert V, Das K. (1994).Vitamin B12 in Modern Nutrition in Health and Disease. 8th ed. Baltimore, MD: Williams \& Wilkins.

3. Institute of Medicine. Food and Nutrition Board. Dietary Reference Intakes: Thiamin, Riboflavin, Niacin, Vitamin B6, Folate, Vitamin B12, Pantothenic Acid, Biotin, and Choline Washington, DC: National Academy Press, 1998.

4. Klee G. G. (2000). Cobalamin and folate evaluation: measurement of methyl malonic acid and homocysteine vs vitamin B (12) and folate. Clin Chem; 46:1277-83.

5. Clarke R. (2008). B-vitamins and prevention of dementia. Proc Nutr Soc; 67:75-81. 
6. Andrès E, Federici L, Affenberger S, Vidal-Alaball J, Loukili NH, Zimmer J, et al. (2007). B12 deficiency: a look beyond pernicious anemia. J Fam Pract; 56:537-42.

7. Fedosov SN. (2010). Metabolic signs of vitamin B (12) deficiency in humans: computational model and its implications for diagnostics. Metab Clin Exp; 59:1124-38.

8. Risch M, Meier DW, Sakem B, et al. (2015). Vitamin B12 and folate levels in healthy Swiss senior citizens: a prospective study evaluating reference intervals and decision limits. BMC Geriatr; 15:82.

9. Solberg HE. (2004). The IFCC recommendation on estimation of reference intervals. The Ref Val program. Clin Chem Lab Med; 42(7):710-4.PMID:15327004.

10. Utah Governor Huntsman recognizes medical laboratory week. Clinical Lab Products. Issue Stories. May 2006

11. Appleton C, Caldwell G, McNeil A et al. (2007). Recommendation for lipid testing and reporting by Australian pathology laboratories. Clin Biochem Review; 28: 32-45.

12. Clinical Laboratory Standards Institute. Defining, Establishing, and Verifying Reference Intervals in the Clinical Laboratory: Approved Guideline. 3rded (C28- A3).Wayne, PA: Clinical Laboratory Standards Institute; 2008.

13. Ceriotti F. (2007). Prerequisites for use of common reference intervals. Clin Biochem Rev; 28:115-121.

14. Reed AH, Henry RJ, Mason WB (1971) Influence of statistical method used on the resulting estimate of normal range. Clinical Chemistry, 17:275-284.

15. Tukey JW (1977) exploratory data analysis. Reading, Mass: Addison-Wesley Publishing Company.

16. https://www.mayocliniclabs.com/testcatalog/Clinical+and+Interpretive/9154. Last accessed: 26, April, 2019. 